\title{
Histomorphological profile of lesions in cystoscopic bladder biopsies - a prospective study in North Maharashtra
}

\begin{abstract}
Introduction: Urinary bladder lesions constitute an important source of clinical signs and symptoms which are more disabling than lethal. The present study aimed to study the frequency of bladder lesions and to study the clinico-histomorphological features of various bladder lesions with special emphasis on grading and staging of the bladder tumours.
\end{abstract}

Materials and methods: The present study is a 3 years prospective study carried out in the department of pathology. All patients who visited to urology outpatient department with lower urinary tract symptoms and obstructive bladder symptoms were subjected to cystoscopy. The detailed clinico-histomorphological features of cystoscopy biopsies are studied using WHO/ISUP 2004 histological grading and TNM staging are used in classifying the bladder tumours.

Results: Out of 139 cystoscopy biopsies, the non-neoplastic bladder lesions constituted $26.6 \%$ and neoplastic lesions constituted $73.38 \%$. Among the neoplastic, benign lesions showed $10.79 \%$ of the cases and malignant lesions showed $62.58 \%$ of the cases. Among the malignant lesions, urothelial carcinoma was the commonest accounting for $55.39 \%$ of the cases with increased prevalence of high grade papillary urothelial carcinoma $(55.43 \%)$ and invasive papillary urothelial carcinoma accounting for $78.25 \%$ of the cases.

Conclusions: The present study has stressed the importance of Histopathological examination with special emphasis on the study of serial sections, inclusion of smooth muscle in the biopsy for accurate grading and staging so as to decrease the mortality and morbidity due to bladder tumours.

Keywords: urothelial lesions, urothelial carcinoma, high grade, low grade
Volume 3 Issue I - 2016

\author{
NV Dravid,' Rajeshwari K,' Karibasappa \\ $\mathrm{GN},{ }^{2}$ Ashish Patil \\ 'Department of Pathology, ACPM Medical College, India \\ ${ }^{2}$ Department of Public Health Dentistry, ACPM Dental College \\ and Hospital, India \\ ${ }^{3}$ Department of Urology, ACPM Medical College, India
}

Correspondence: Rajeshwari K, Assistant Professor, Department of Pathology ACPM Medical College, Dhule,Maharashtra, India, \# 8 staff quarters, Tel 91750768899 , Email ujwalgk@gmail.com

Received: September 23, 2016 | Published: October 21, 2016
Abbreviations: OPD, outpatient department; TURBT, transurethral resection of bladder tissue; LUTS, lower urinary tract symptoms; PUNLMP, papillary urothelial neoplasm of low grade malignant potential; PAS, periodic acid- schiff; JMS, jones' methenamine siver stain; PEM, periodic acid-siver methenamine; GMS, gomori's trichrome stain; MTS, massons trichrome stain; IHC, immunohistochemistry

\section{Introduction}

In this modern era, there have been increased prevalence of bladder lesions including both neoplastic and non neoplastic lesions and have been contributed for significant disabling than lethal. Bladder tumours constitute one of the most common urologic condition and accounts for significant morbidity and mortality. ${ }^{1,2}$ It is the second most common neoplasm of genitourinary tract next to prostate and sixth most common cancer in the world wide. ${ }^{3}$ These neoplasms of bladder pose biologic, clinical, diagnostic and therapeutic challenges to both urologist and uropathologist, as these bladder tumours represent a heterogeneous group of tumours with different subtypes and behavioral patterns. ${ }^{4,5}$ As per the data of Indian cancer registry, it is the ninth most common cancer and accounts for $3.9 \%$ of all cancers. ${ }^{6}$ Since most of the bladder tumours are known for its recurrence, rapid progression to high grade and stage and it presents with various non- specific symptoms for which there is a need for accurate diagnostic tests. The significant diagnostic test includes cystoscopy, bladder biopsy and urine cytology for accurate diagnose, management and prognostic assessment of the patient.

Giving the importance of bladder lesions in genitor urinary pathology, the present study was undertaken to study the clinicopathological features of bladder lesions with special emphasis on grading and TNM staging, so as to prevent the mortality and morbidity associated with urinary bladder carcinoma.

\section{Materials and methods}

The present study is a prospective type carried out in the department of pathology over a period of three years between June 2012-June 2015 and included 139 cases in the study.

All the patients who visited the urology outpatient department (OPD) with lower urinary tract symptoms and obstructive urinary symptoms were included in the study. After the informed written consent of the patients, fiberoptic cystoscopy bladder biopsies were taken from the walls of the bladder. The cystoscopic bladder biopsies taken were fixed in $10 \%$ buffered formalin and then processed with embedding in paraffin wax. Four microns thick sections were taken and stained with regular haematoxylin and eosin stain ( $\mathrm{H}$ and $\mathrm{E})$. The study of biopsy specimens along with the histomorphological 
changes under low and high power magnification were studied by light microscope. The WHO/ISUP-2004 grading system was used to grade the bladder neoplasms ${ }^{7}$ and staging was done according to TNM staging. ${ }^{7}$

Inclusion criteria: All the transurethral resection of bladder tissue (TURBT) biopsies received in the department of pathology.

Exclusion criteria: Autolysed and inadequate biopsies were excluded.

\section{Results}

Out of total 139 cases, 71 cases $(51.1 \%)$ were males and 68 cases $(48.9 \%)$ were females with peak age incidence is between 60-69years as shown in Graph 1. In the study, we found that the majority of the patients with benign lesions presented with lower urinary tract symptoms (LUTS) accounting for $82.7 \%$ of the cases and those with malignant lesions present with haematuria accounting for $90.8 \%$ of the cases as shown in Graph 2. The details of the histomorphological diagnosis of bladder lesions are given in table 1. In our study, we observed that majority of the bladder lesions were malignant constituting $62.58 \%$ (87cases) of the cases, among them the most common we found was urothelial carcinoma followed by squamous cell carcinoma and adenocarcinoma constituting 55.39\%, 5.03\% and $2.15 \%$ respectively. As per histological grading WHO/ISUP (2004) used in the study we found high prevalence of high grade urothelial carcinoma followed by low grade urothelial carcinoma, urothelial papilloma and papillary urothelial neoplasm of low grade malignant potential (PUNLMP) accounting for $55.43 \%, 22.82 \%, 16.3 \%$ and $5.43 \%$ respectively as shown in Table 2 .

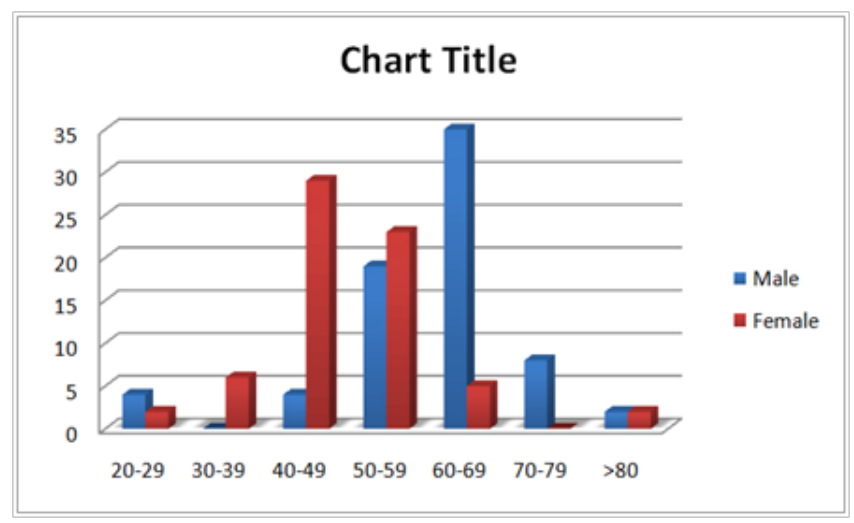

Graph I Age and gender wise distribution of bladder lesions.

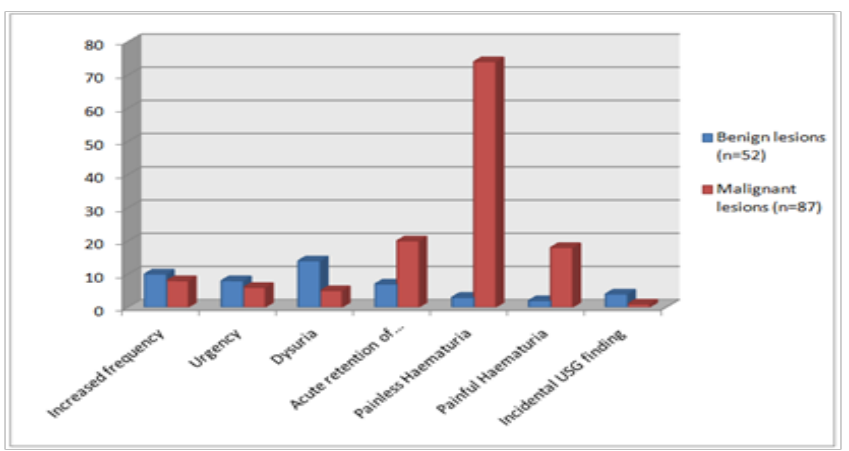

Graph 2 Clinical presentations of the patients with bladder lesions.
Table I Histomorphological diagnosis of bladder lesions

\begin{tabular}{llll}
\hline SI. No & Histomorphological diagnosis & No. of cases & Percentage \\
\hline I & Non neoplastic & 52 & $37.41 \%$ \\
1 & Non-specific cystitis & 18 & $12.94 \%$ \\
2 & Follicular cystitis & 6 & $4.31 \%$ \\
3 & Eosinophilic cystitis & 1 & $0.71 \%$ \\
4 & Cystitis glandularis & 2 & $1.43 \%$ \\
5 & Cystitis cystica & 1 & $0.71 \%$ \\
6 & Squamous metaplasia & 8 & $5.75 \%$ \\
7 & Tubercular cystitis & 1 & $0.71 \%$ \\
II & Neoplastic & 102 & $73.38 \%$ \\
a. & Benign & & \\
& Urothelial papilloma & 15 & 10.79 \\
b. & Malignant & 87 & $62.58 \%$ \\
1 & Urothelial carcinoma & 77 & $55.39 \%$ \\
2 & Squamous cell carcinoma & 7 & $5.03 \%$ \\
3 & Adenocarcinoma & 3 & $2.15 \%$ \\
& Total & 139 & $100 \%$ \\
\hline
\end{tabular}

Table 2 Histological grading of urothelial neoplasm as per isup/Who 20047

\begin{tabular}{llll}
\hline S1. No & Grade & No. of cases & Percentage \\
\hline 1. & Papilloma & 15 & $16.3 \%$ \\
2. & PUNLMP & 5 & $5.43 \%$ \\
3. & $\begin{array}{l}\text { Low grade papillary urothelial } \\
\text { carcinoma }\end{array}$ & 21 & $22.82 \%$ \\
4. & $\begin{array}{l}\text { High grade papillary urothelial } \\
\text { carcinoma }\end{array}$ & 51 & $55.43 \%$ \\
& Total & $\mathbf{9 2}$ & $\mathbf{1 0 0 \%}$ \\
\hline
\end{tabular}

*PUNLMP, papillary urothelial neoplasm of low grade malignant potential.

In our study, we made an attempt to evaluate the pattern of tumor growth, its degree of differentiation, progression and associated mucosal changes. In 15 cases of urothelial papilloma, it is characterized by discrete papillary growth with a central fibrovasular core lined by urothelium of normal thickness and cytology (Figure 1). In five cases of Papillary urothelial neoplasm of low grade malignant potential (PUNLMP), histopathologically the tumor is characterized by delicate, orderly, tenuous papillary structures with orderly arrangement of cells within the papillae with minimal architectural abnormalities and nuclear atypia usually limited to basal layer irrespective of cell thickness (Figure 2). The major distinction from papilloma is that in PUNLMP the urothelium is much thicker and nuclei are significantly enlarged. In 21 cases of low grade urothelial carcinoma, histologically it is characterized with papillary axes which are more compact, crowded, fused at the base and lined by unordered cells showing both architectural and cytological abnormalities with frequent mitosis (Figure 3). In 51 cases of high grade urothelial carcinoma showed fused papillary axes over the large areas resulting in sheets and solid areas. The cells have enlarged, hyperchromatic, pleomorphic nucleus in full thickness of the epithelium with increased atypical mitosis (Figure 4). As per TNM staging used in our study we observed 
majority of the bladder tumours were invasive accounting for $78.25 \%$ (Figure 5 \& Figure 6) as compared to non-invasive bladder tumours constituting $21.73 \%$ of the cases as shown in Table 3 .

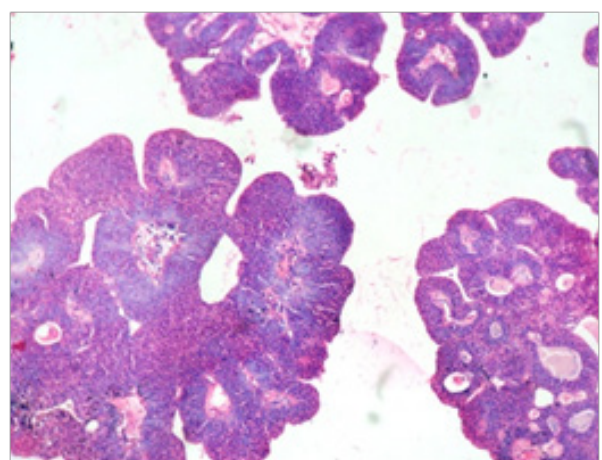

Figure I Urothelial papilloma showing discrete papillae lined by normal thickened urothelium with normal cytology ( $\mathrm{H}$ and $\mathrm{E}$ stain, I00X).

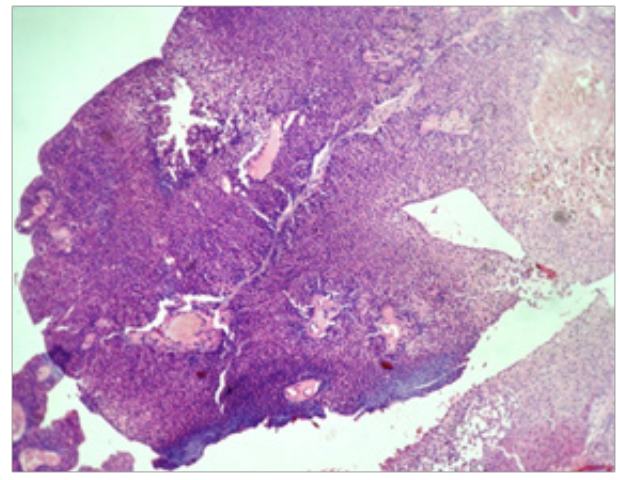

Figure 2 Papillary urothelial neoplasm of low malignant potential shows delicate papillae lined by hyperplastic urothelium with minimal nuclear atypia ( $\mathrm{H}$ and $\mathrm{E}$ stain, I00X).

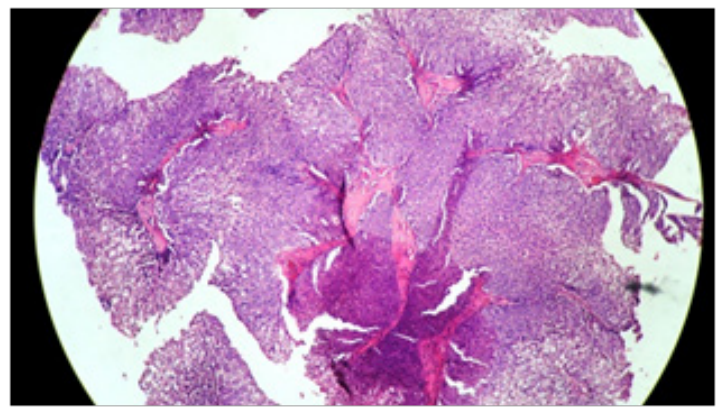

Figure 3 Low grade papillary urothelial carcinoma shows compact, crowded papillae lined by hyperplastic urothelium with moderate nuclear atypia $(\mathrm{H}$ and E stain, I00X).

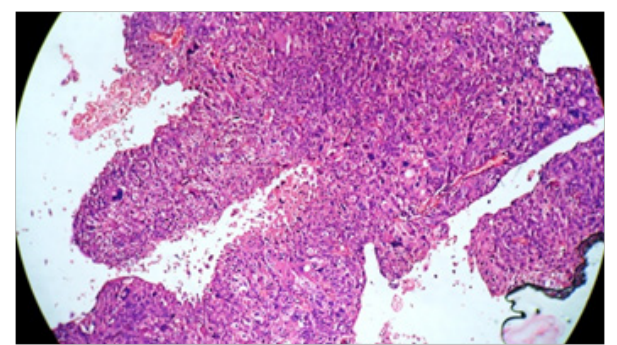

Figure 4 High grade papillary urothelial carcinoma shows tumour cells in sheets and solid areas with marked cellular and nuclear atypia increased mitosis. ( $\mathrm{H}$ and $\mathrm{E}$ stain, 400X).

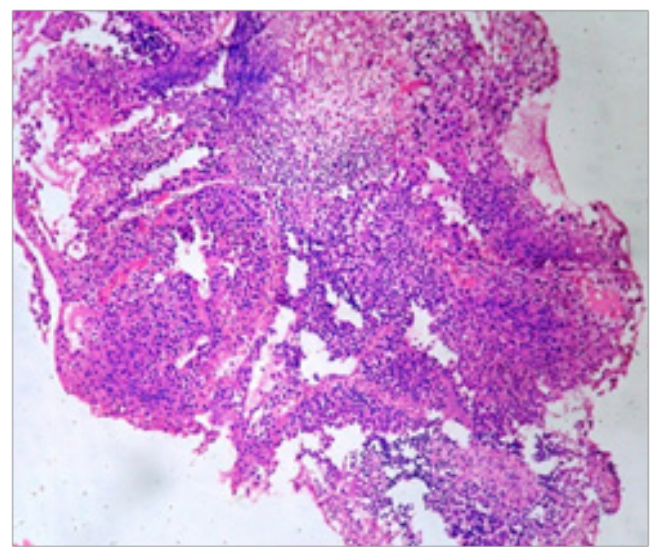

Figure 5 Stage $\mathrm{PTI}$ showing tumour cells in clusters and singly infiltrating into the lamina propria. ( $\mathrm{H}$ and $\mathrm{E}$ stain, 400X).

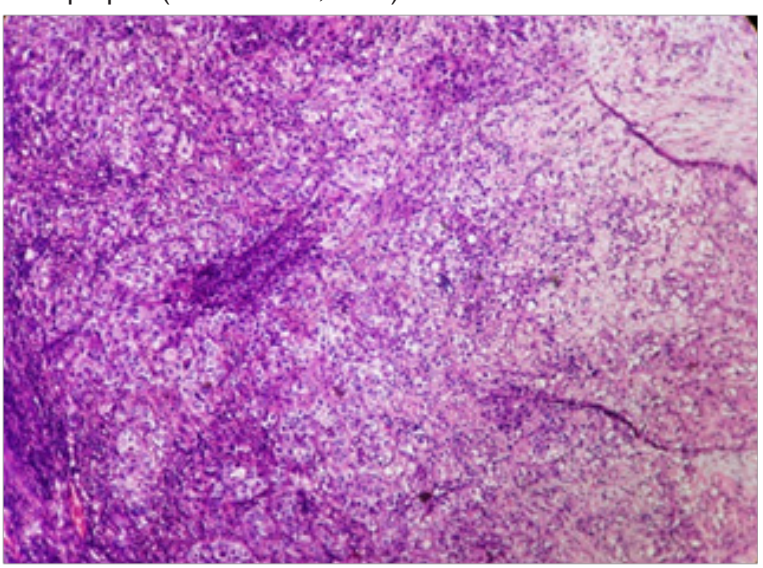

Figure 6 Stage pT2 showing tumour cells infiltrating into the detrusor muscle bundles. ( $\mathrm{H}$ and $\mathrm{E}$ stain, 400X).

Table 3 Staging Of urothelial neoplasm as per Tnm staging7

\begin{tabular}{llll}
\hline Sl. No & Staging & $\begin{array}{l}\text { No. of } \\
\text { cases }\end{array}$ & Percentage \\
\hline 1. & pTa(Confined to epithelium) & 20 & $21.73 \%$ \\
2. & pT1 (Invasion into lamina propria) & 49 & $53.26 \%$ \\
3. & pT2 (Invasion into superficial & 18 & $19.56 \%$ \\
4. & muscle) & 3 & $3.26 \%$ \\
5. & pT3 (Invasion into deep muscle) & 2 & $2.17 \%$ \\
& Total & $\mathbf{9 2}$ & $\mathbf{1 0 0 \%}$ \\
\hline
\end{tabular}

\section{Discussion}

The present study is undertaken mainly to highlight the importance of histopathological examination in the diagnosis of bladder lesions. In recent days, the diagnosis and monitoring of bladder lesions are made by combination of cystoscopy, histopathology and urine cytology. ${ }^{8}$ All these diagnostic methods have its own limitations and cannot diagnose the presence of bladder tumours at every point of time. ${ }^{9}$ Advantages of cystoscopy are 1) helps in localizing the bladder tumours. 2) Detects low grade papillary lesions. Disadvantages are 1) Cystoscopy cannot visualize all the areas of bladder 2) Certain 
tumours like carcinoma insitu cannot be detected by cystoscopy. 3) It cannot comment on tumour grade and its depth of invasion.

Hence histopathology remains the gold standard method for diagnostic and therapeutic purpose. But it has its own limitations due to friability nature of bladder tumours, there may be technical impediments and interpretation errors like electro coagulation artifacts, retraction artifacts, exclusion of smooth muscle fibers from the mucosa pose difficulties in the histological grading and staging of the tumour. In such situations these limitations can be overcome by studying, multiple serial sections, by inclusion of smooth muscle during cystoscopic biopsy and by the usage of special stains to ensure accurate and definitive diagnosis.

Various special stains which includes histochemical and immunohistochemical stains are used

i. Gram stain, Zheil - Neelson stain, Periodic Acid- Schiff (PAS) Jones' methenamine Siver stain (JMS) are used to differentiate granulomatous lesions that mimics carcinoma like infections of tuberculosis, malakoplakia, cryptococcus, mucormycosis and schistosomia haematobium infestation.

ii. To differentiate other tumour like conditions mimicking carcinoma like inflammatory myofibroblastic tumour, pseudosarcomatous stromal reaction, pseudocarcinomatous proliferation, fibroepithelial polyps, collagen polyps, hamartoma etc can be differentiated that mimics carcinoma.

iii. Using special stains like Periodic Acid- Schiff (PAS), Periodic Acid-Siver methenamine (PEM) and Jones' methenamine Siver stain (JMS) are used for delineation of basement membrane and is used for detection of early invasive urothelial carcinoma.

iv. Gomori's trichrome stain (GMS), Massons Trichrome stain (MTS) are used for staining of smooth muscle and collagen like laminin which are usually advocated for detection of early stromal invasion.

v. Histochemical stains for tenascin, an extra cellular matrix protein is strongly positive in invasive high grade carcinoma.

vi. Other stains like AgNOR, and $\mathrm{Ki}-67$ are used as proliferative markers that can differentiate between premalignant and malignant lesions of bladder.

vii. Immunohistochemical stains like CK-20, CK7, CK5/6, p53, and p63 stains are used as adjuvant for diagnosis of urothelial carcinoma.

In the era of modernization, robust techniques like flow cytometry, chromosomal analysis, genomics, proteomics and immunohistochemistry (IHC) applications are used for an accurate classification and prognostication of bladder tumours. ${ }^{10}$ But these techniques are not routinely used due to lack of specificity, sensitivity and also due to exorbitant cost and inaccessibility to common people.

In our study, we have utilized all the three diagnostic modalities but emphasizing more on the Histopathological diagnosis with respect to histological grading and staging of bladder tumours. We selected, WHO/ISUP (2004) classification for histological grading in the present study due to its various advantages like 1) Uniform terminology and common definition used and accepted by various urological pathologist. 2) Well defined criteria for preneoplastic and neoplastic bladder tumours leading to greater inter-observer reproducibility. 3) Terminologies of WHO/ISUP (2004) classification and terminologies of urine cytology are similar facilitating better cyto-histologic correlation. 4) Prevents over or under diagnosis of urothelial tumours. 5) Identification of distinct group of patients with high grade papillary urothelial carcinoma and carcinoma insitu for intravesical therapy. 6) Removes ambiguity in diagnostic categories. 7). It mainly shows prognostically significant categories. 8) It also gives idea regarding the recurrence rate, progression grade and stage and survival rates between tumours of different categories of noninvasive papillary urothelial neoplasms of bladder as given in table 4. ${ }^{11}$

In our study we observed that bladder lesions are common between the age group of 60-69years constituting $48.64 \%$ of the cases and is in concordance with Vaidya et al. ${ }^{12} \&$ Matalka et al. ${ }^{13}$ The bladder lesions showed male preponderance than female accounting for $51.1 \%$ and $48.9 \%$ of the cases respectively. Our observations are in accordance with Lim et al. ${ }^{14}$ \& Vaidya et al. ${ }^{12}$ The increased prevalence of bladder lesions in males observed in our study could be attributed to cigarette smoking, industrial exposure to acrylamine, schistosoma haematobium, cyclophosphamide, artificial sweetners and long acting analgesics. Various literatures state that the exact mechanism of inducing the cancer by these risk factors is unclear but a number of cytogenetic and molecular alterations are heterogenous. ${ }^{15}$

In the present study majority of the bladder lesions noted were malignant lesions accounting for $62.58 \%$ of the cases and correlated with the Vibhav KG et al study $(96.87 \%)$ and Vaidya $\mathrm{S}$ et al study $(77.57 \%))^{16,12}$ We have also observed that, among the malignant lesions of bladder most common was urothelial carcinoma followed by squamous cell carcinoma and adenocarcinoma accounting for $55.39 \%, 5.03 \%$ and $2.15 \%$ respectively and is in accordance with Mahesh K et al. ${ }^{17}$ and discordance with Mohammad M et al (93.3\%) and Vibhav KG et al. $(93 \%)^{8,16}$ as shown in Table 5.

We have found a wide spectrum of bladder lesions of which $26.6 \%$ of the cases accounted for non neoplastic lesions of bladder and $73.38 \%$ of the cases accounted for neoplastic lesions. This wide spectrum of bladder lesions in our study could be attributed to, free screening camps conducted by our centre. Our centre being one of the largest where free cystoscopic bladder biopsies were carried out in suspected patients presenting with obstructive and lower urinary tract symptoms.

Present study showed increased prevalence of high grade urothelial carcinoma accounting for $55.43 \%$ and is in concordance with Vibhav $\mathrm{KG}$ et al. ${ }^{16}$ and Vaidya $\mathrm{S}$ et al, ${ }^{12}$ Mahesh K et al. ${ }^{17}$ and Satya et al. ${ }^{18}$ as shown in Table 6 .

Our study showed increased prevalence of invasive urothelial carcinoma than non-invasive urothelial carcinoma and correlated with Christopher et al. ${ }^{19}$ and Vaidya et al. ${ }^{12}$ as shown in Table 7. The increased prevalence of high grade urothelial carcinoma in our study may be due to lack of awareness among the people, low socioeconomic status and poor hygienic practices among the patients have contributed for the same.

In our study the diagnosed cases of invasive urothelial carcinoma were treated surgically with radical cystectomy with urinary diversion. Preoperative radiotherapy and adjuvant chemotherapy was given for patients with localized disease in urothelial carcinoma. Patients with 
squamous cell carcinoma and adenocarcinoma were treated with of the case. radiotherapy. On follow-up patient doing well till the date of review

Table 4 Comparison of recurrence rate, grade and stage progression and survival rates of non-invasive papillary urothelial neoplasms. ${ }^{\prime \prime}$

\begin{tabular}{lllll}
\hline & Papilloma & $\begin{array}{l}\text { Papillary neoplasm of low grade } \\
\text { malignant potential }\end{array}$ & $\begin{array}{l}\text { Low grade papillary } \\
\text { urothelial carcinoma }\end{array}$ & $\begin{array}{c}\text { High grade papillary } \\
\text { urothelial carcinoma }\end{array}$ \\
\hline $\begin{array}{l}\text { Recurrence } \\
\begin{array}{l}\text { Grade } \\
\text { progression }\end{array}\end{array}$ & $0-8 \%$ & $27-47 \%$ & $48-71 \%$ & $55-58 \%$ \\
$\begin{array}{l}\text { Stage progression } \\
\text { Survival }\end{array}$ & $0 \%$ & $11 \%$ & $7 \%$ & Not applicable \\
\end{tabular}

Table 5 Comparison of prevalence of various neoplastic lesionso bladder in different studies

\begin{tabular}{|c|c|c|c|c|}
\hline Neoplastic lesions & Mahesh K et al. ${ }^{17}$ & Mohammad M et al. ${ }^{8}$ & Vibhav KG et al. ${ }^{16}$ & Present study \\
\hline Urothelial carcinoma & $28(46.6 \%)$ & $467(93.3 \%)$ & $93(93 \%)$ & $77(53.39 \%)$ \\
\hline Squamous cell carcinoma & $2(3.33 \%)$ & $13(2.6 \%)$ & $2(2 \%)$ & $7(5.03 \%)$ \\
\hline Adenocarcinoma & $2(3.33 \%)$ & $10(2 \%)$ & $1(1 \%)$ & $3(2.15 \%)$ \\
\hline
\end{tabular}

Table 6 Comparison of prevalence of different histological grades of bladder neoplasms in various studies

\begin{tabular}{|c|c|c|c|c|c|c|}
\hline Grading & Laishram et al. ${ }^{6}$ & Mahesh K et al. ${ }^{17}$ & Vaidya et al. ${ }^{12}$ & Satya et al. ${ }^{18}$ & Vibhav KG et al. ${ }^{16}$ & Present study \\
\hline PUNLMP & $7.69 \%$ & - & $10.28 \%$ & - & $4.10 \%$ & $5.43 \%$ \\
\hline LGPUC & $53.85 \%$ & $42.85 \%$ & $29.91 \%$ & $25.00 \%$ & $32.29 \%$ & $22.82 \%$ \\
\hline HGPUC & $34.61 \%$ & $53.57 \%$ & $32.70 \%$ & $62.85 \%$ & $60.41 \%$ & $55.43 \%$ \\
\hline
\end{tabular}

*PUNLMP, Papillary urothelial neoplasm of low grade malignant potential;

*LGPUC, Low grade papillary urothelial carcinoma.

*HGPUC, High grade papillary urothelial carcinoma.

Table 7 Comparison of prevalence of different stages of bladder neoplasms in various studies

\begin{tabular}{lllll}
\hline TNM stage & Christopher et al. ${ }^{19}$ & Laishram et al. & Vaidya et al. ${ }^{\mathbf{1 2}}$ & Present study \\
\hline pTa & $23(33.3 \%)$ & $14(53.85 \%)$ & $39(48.14 \%)$ & $20(21.73 \%)$ \\
pT1 & $15(20.0 \%)$ & $4(15.38 \%)$ & $18(22.22 \%)$ & $49(53.26 \%)$ \\
pT2 & $8(10.7 \%)$ & $8(30.77 \%)$ & $24(29.63 \%)$ & $18(19.56 \%)$ \\
pT3 & $9(12.0 \%)$ & & $3(3.26 \%)$ \\
pT4 & $14(18.7 \%)$ & & $2(2.17 \%)$ \\
\hline
\end{tabular}

\section{Conclusion}

We conclude that urinary bladder lesions are common in north Maharashtra. A high prevalence of $62.58 \%$ of the cases of bladder tumours is noted. The early and definitive diagnosis with accurate grading and staging of bladder tumours can be done by histopathology. The importance of including the smooth muscle in the biopsy, study of multiple serial sections and with usage of special stains needs to be emphasized in definitive diagnosis of bladder neoplasms. Attempts should also be made to create awareness among the public regarding the bladder symptoms so as to prevent mortality and morbidity faced by the people due to advanced bladder tumours.

\section{Acknowledgements}

None.

\section{Conflict of interest}

The author declares no conflict of interest.

\section{References}

1. Jemal A, Siegel R, Ward E, et al. Cancer statistics. CA Cancer J Clin. 2009;59:225-249.

2. Epstein JI. The lower urinary tract and male genital system. In: Kumar $\mathrm{V}$, et al. editors. Robbins and Cotran pathologic basis of diseases. 7 th ed. Saunders, USA; 2004. p. 1023-1058.

3. Al-Samawi AS, Aulaqi SM. Urinary bladder cancer in Yemen. Oman Med J. 2013;28(5):337-340.

4. Kumar V, Abbas AK, Fausto N. The lower urinary tract and male genital system. Robbins and Cotran pathologic basis of diseases. 7 th ed. Saunders, Philadelphia, USA; 2004. p.1026-1036. 
5. Hussain N, Shumo A, Mekki S, et al. A clinicopathological study of urinary bladder neoplasms in patients at three centers in Khartoum, Sudan. Sudan Journal of medical science. 2009;4(3):249-255.

6. Laishram RS, Kipgen P, Laishram S, Khuraijam S, Sharma DC (2012) Urothelial tumours of the urinary bladder in Manipur: A histopathological perspective. Asia Pacific J Cancer Prev 13(6): 2477-2479.

7. Eble JN, Sauter G, Epstein JI, et al. Tumours of the urinary system. WHO classification of tumours- Pathology and genetics of tumours of the urinary system and male genital organs. Lyon, France: IARC Press; 2004 p. $89-158$.

8. Muhammed M, Javed IK, Altaf H, et al. Urinary bladder tumours in sourthern Pakistan: A histopathological perspective. MJC. 2014;5(3):167173.

9. Reuter VE. The pathology of bladder cancer. Urology. 2006;67(3 Suppl):11-18.

10. Mubarak M. A step towards refining prognostication in individual patients with) A step towards refining prognostication in individual patients with bladder cancer. Urol Ann. 2013;5(2):85-87.

11. Mahul B Amin. AP110 Urinary bladder biopsy interpretation Part 1. USA: College of American Pathologists; 2004. p. 1-11.

12. Vaidya S, Lakhey M, Sabira KC, et al. Urothelial tumours of the urinary bladder: A Histopathological study of cystoscopic biopsies. JNMA J Nepal Med Assoc. 2013;52(191):475-478.
13. Matalka I, Bani-Hani K, Shotar A, et al. Transitional cell carcinoma of the urinary bladder: a clinicopathological study. Singapore Med J. 2008;49(10):790-794.

14. Lim M, Adsay NV, Grignon D, et al. Urothelial carcinoma with villoglandular differentiation: a study of 14 cases. Mod Pathol. 2009;22(10):1280-1286.

15. Hasan SM, Imtiaz F, Hasan SM. Frequency of transitional cell carcinoma in local suburban population of Karachi. JLUMHS. 2007. p. 83-85.

16. Vaibhav KG, Surendra PV, Dharm CK. Spectrum of lesions in urinary bladder biopsies: Histopathological study. Int $J$ Dent Med Res. 2015;1(6):42-46.

17. Mahesh K, BR Yelikar. Spectrum of lesions in cystoscopic bladder biopsies- A histopathological study. Al Ameen J Med Sci. 2012;5(2):132136.

18. Satya M, Chinnaswamy P. Urinary bladder cancer: A clinicopathological and histological study. J Med Sci. 2014;14:206-209.

19. Christopher $\mathrm{HCH}$, Praveen S, Goh EH, et al. Clinicopathological features of bladder tumours in a single institution in Malaysia. Asian Pac J Cancer Prev. 2010;11(1):149-152. 